\title{
No. 68-144
}

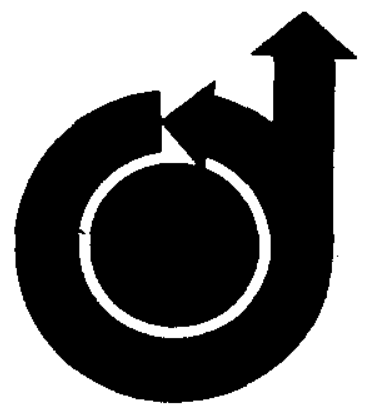

\section{DYNAMIC RESPONSE OF FINITE LENGTH CYLINDRICAL SHELLS TO NEARLY UNIFORM RADIAL IMPULSE}

by

I. K. MclVOR and E. G. LOVELL

University of Michigan

Ann Arbor, Michigan

AlAA Paper

No. 68-144
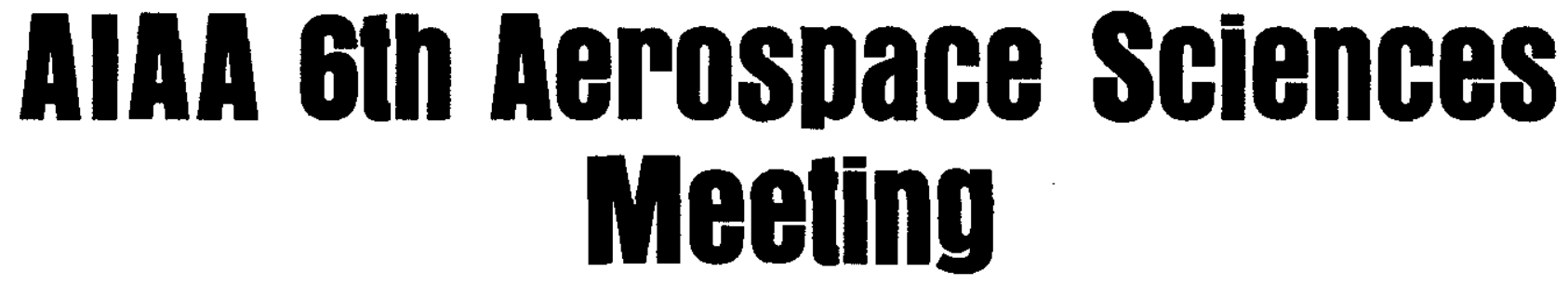

NEW YORK, NEW YORK / JANUARY 22-24, 1968

first publication rights reserved by American Institute of Aeronautics and Astronautics, 1290 Avenue of the Americas, New York, N. Y. 10019. Abstracts may be published without permission if credit is oiven to outhor and 10 AlAA. (Price-AlAA Member $\$ 1.00$, Nonmember $\$ 1.50$ ) 


\title{
DYNAMIC RESPONSE OF FINITE LENGTH CYLINDRICAL SHELLS TO NEARLY UNIFORM RADIAL IMPULSE
}

\author{
I. K. Mclvor* and E. G. Lovell ** \\ The University of Michigan \\ Ann Arbor, Michigan
}

\begin{abstract}
A.bstract
The response of a cylindrical shell to a uniform radial impulse is a simple harmonic motion in which the cross section remains circular. In the presence of small nonuniformities in the imiulse, this motion may be unstable. The instability is characterized by the parametric excitation of flexural modes which exchange energy with the basic motion in a cyclic manner. The stability criterion derived here for finite length cylinders shows this instability may occur over a wide range of shell geometries. The flexural modes which exhibit significant growth are identified. With this the finite time response of the shell is obtained by numerical integration of the nonlinear equations. The nonlinear response is ciominated by a few flexural modes which initially have high growth rates. The amplificatiori of these modes results in displacements and strusses considerably in excess of those associated with the unperturbed response. Finally the imperfection sensitivity of the nonlinear mo: ion is examined.

\section{Introduction}

When shell structures are subjected to time derendent loadings, the dynamic response may be affected by parametric instabilities. A particular class of such instabilities occur in shells. subjected to impulsive loads. The instabilities arise from nonlinear coupling between possible motes of free vibration. As an example, the $r$ exponse of a cylindrical shell to a uniform radial impulse is a simple harmonic motion in which the cross section remains circular, periodically expanding and contracting. But this basic response may be unstable in the presence of small noraniformities in the initial impulse. A number of ! redominantly flexural modes can be parametrically excited to rather large amplitudes. Entrgy is extracted from the initial or basic response and cyclically exchanged with the modes exhibiting significant growth.
A response of this type was obtained for the cylindrical shell by Goodier and McIvor [1]. The investigation was restricted to the case of plane strain, i.e, requiring generators of the shell to remain straight and parallel to the axis. A study of the analogous problem for the axisymmetric spherical shell by Mclvor and Sonstegard [2] produced similar results. In the present paper axial motion and variation along the generators are permitted, and the effect of the length of the shell upon the response is determined.

The related problem of a simply supported cylindrical shell subjected to a suddenly applied pressure has been considered by Bieniek, Fan, and Lackman [3] using Galerkin's method. From a short-term analysis, the growth of displacement perturbations was predicted. The long-term motion was not analyzed. For the present problem of the motion of a finite length cylindrical shell following impulsive pressure, a criterion for the stability of the basic response is obtained and its dependence upon the shell geometry is established. In addition those flexural modes which may exhibit significant growth are identified. With the critical modes determined, the nonlinear equations are integrated numerically to obtain the finite time response. A number of examples are discussed which serve to illustrate the magnification of stresses and displacements associated with the finite time response. The examples also serve to delineate the perturbation sensitivity of the response.

\section{Method of Solution}

The cylindrical shell considered is shown in Figure 1. The constants denoting the length, thickness and midsurface radius are $\ell, h$, and $a$, respectively. The coordinates in the longitudinal, circumferential and radial directions are $x, \theta$, and $z$, and the corresponding displacement components of a point on the midsurface are $u, v, w$. The displacements are considered as functions of the coordinates of a point on the undeformed midsurface $(x, \theta)$ and time t.

\footnotetext{
* Associate Professor of Engineering Mechanics, The University of Michigan.
}

*** Formerly Instructor, The University of Michigan. Now NASA Postdoctoral Fellow, Langley Research Center, Hampton, Virginia.

\footnotetext{
**** Numbers in brackets refer to the bibliography.
} 
To investigate the motion following impulsive pressure, we express the total energy of the shell as is function of the displacements and their spatial and time derivatives. Series representations are cho:ien for the displacements which satisfy given boundary conditions. After these representations are introduced into the energy functional, the time dep.ndent coefficients can be considered as generalized coordinates. Thus they are solutions of the as sociated Lagrange equations.

In deriving the energy functional appropriate for the ensuing stability analysis, it is necessary to retain quadratic terms in the strain-displacement relations. But in the motion of thin shells folluwing radial loading, the tangential components of the displacement are generally small compared to the radial component. Thus quadratic terms involving only $w$ and its derivatives are retained. With this and using the usual as sumptions of thin shell theory as to conservation of normals, unclianging thickness coordinates, and neglecting $\left(\mathrm{h} / \mathrm{iz}^{2}\right)^{2}$ compared to unity, an appropriate energy functional is obtained. It is derived in detail in [4], and is given for reference in the appendix.

The displacement representation used in the present analysis is

$$
\begin{aligned}
& u=\sum_{m=0}^{\infty} \sum_{n=0}^{\infty} U_{m n}(t) \sin \frac{m \pi x}{l} \cos n \theta \\
& \because=\sum_{m=0}^{\infty} \sum_{n=0}^{\infty} v_{m n}(t) \cos \frac{m \pi x}{l} \sin \theta \\
& v=\sum_{m=0}^{\infty} \sum_{n=0}^{\infty} w_{m n}(t) \cos \frac{m \pi x}{l} \cos n \theta
\end{aligned}
$$

At wach boundary of the shell the slope $\partial w / \partial x$ and the axial displacement vanish, whereas the radial and circumferential displacements do not. The effective radial and circumferential shear per unit length are zero at the boundaries. Although these conditions ar re possibly of less physical interest than more common geometric constraints, they give $r$ ise in the present problem to considerable simplification of the energy expression. Thus they permit investigation of the effect of finite length on the stability analys is without undue algebraic complication.

The time dependent coefficients in (1) are considered as generalized coordinates. The associated Lagrange equations are listed in the Appendix. 'This highly coupled, infinite system of differential equations govern the free nonlinear motion of the shell. Reasonable approximations must be made before useful solutions can be obtiained.

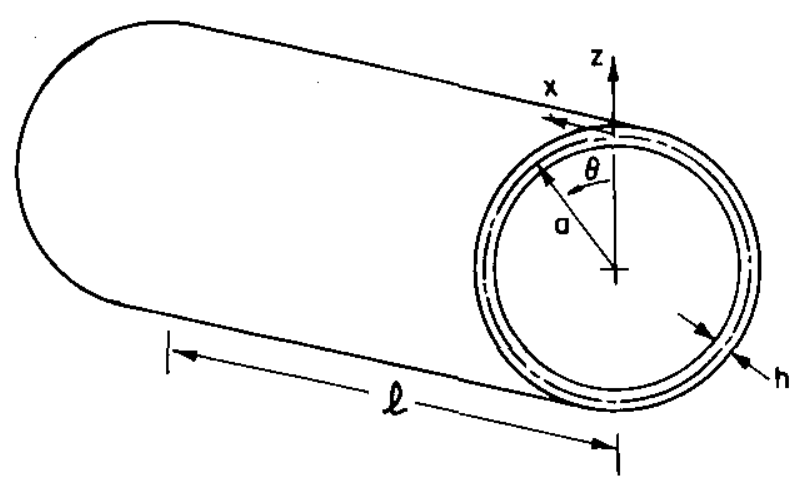

Fig. I Shell Geometry

Initial Motion - Stability of the Basic Response

If the impulsive pressure is of sufficiently short duration, its effect is to impart an initial, radial velocity to the shell. For a perfectly uniform distribution $W_{O O}$ is the only nonzero term in the representation (1). From $(A-6)$ the governing equation is

$$
\ddot{w}_{0}+\dot{w}_{0}=0
$$

where the dot denotes differentiation with respect to dimensionless time $T$ defined as

$$
T=\mathrm{ct} / \mathrm{a}, \quad \mathrm{c}^{2}=\mathrm{E} / \rho\left(1-v^{2}\right)
$$

The associated initial conditions are

$$
w_{00}(0)=0, \quad \dot{w}_{00}(0)=a v_{0} / c
$$

where $v_{0}$ is the uniform velocity imposed on the shell. For elastic motion $v_{0}<c$. The basic response is

$$
w_{00}=\frac{a v_{0}}{c} \sin T
$$

To investigate the stability of the basic response we perturb the motion by considering a small nonuniformity in the initial velocity distribution. The response is now given by (1) with $W_{\text {oo }}$ being the basic response as affected by the perturbation and the remaining terms representing the perturbational response. The nonzero initial conditions associated with equations $(A-6)$ and $(A-7)$ are

$\dot{\mathrm{w}}_{\mathrm{oO}}(0)=\mathrm{av}_{\mathrm{o}} / \mathrm{c}, \quad \dot{\mathrm{w}}_{\mathrm{mn}}(0)=\epsilon_{\mathrm{mn}} \mathrm{av_{0 }} / \mathrm{c}$

where $\epsilon_{m n}$ is much less than unity for all pairs of $m$ and $n$ except $(0.0)$.

The summations in $(A-6)$ and $(A-7)$ involve products of coefficients arising from the nonuniformity. For small values of time they are of perturbational magnitude and may be neglected. 
Witis this $W_{00}$ is the basic response (5), and equations $(\mathrm{A}-7)$ reduce to

$$
\begin{aligned}
& \ddot{U}_{m n}+C_{1} U_{m n}+C_{2} v_{m n}+C_{3} w_{m n}=0 \\
& \ddot{v}_{m n}+C_{4} v_{m n}+C_{2} U_{m n}+C_{5} w_{m n}=0
\end{aligned}
$$

$$
\begin{aligned}
& \ddot{w}_{m n}+c_{3} U_{m n}+c_{5} v_{m n} \\
& +w_{m n}\left\{c_{6}+\frac{w_{0 o}}{a}\left[n^{2}+\frac{m^{2} \pi^{2}}{3}\left|\frac{a}{l}\right|^{2}\right]\right\}=0
\end{aligned}
$$

whe re

$$
\begin{gathered}
C_{1}=\frac{n^{2}}{3}+\pi^{2} m^{2}\left(\frac{a}{l}\right)^{2} \\
\dot{C}_{2}=\frac{2 \pi m n}{3} \frac{a}{l} \\
C_{3}=\frac{\pi m}{3} \frac{a}{l}+\frac{\pi^{3} m^{3}}{3}\left(\frac{h}{a}\right)^{3}\left(\frac{a}{l}\right)^{3} \\
-\frac{\pi m n^{2}}{36}\left(\frac{h}{a}\right)^{2} \frac{a}{l} C_{4} \pm n^{2}+\frac{\pi^{2} m^{2}}{3}\left(\frac{a}{l}\right)^{2} \\
\left.C_{5}=n+\frac{\pi^{2} m^{2} n^{9}}{9}\left(\frac{h}{a}\right)^{2} \mid \frac{a}{l}\right)^{2} \\
C_{6}=1+\frac{\pi^{4} m^{4}}{12}\left|\frac{h}{a}\right|^{2}\left(\frac{a}{l}\right)^{4}+\frac{n^{4}}{l 2}\left(\frac{h}{a}\right)^{2} \\
-\frac{n^{2}}{6}\left(\frac{h}{a}\right)^{2}+\frac{\pi^{2} m^{2} n^{2}}{6}\left(\frac{h}{a}\right)^{2}\left(\frac{a}{l}\right)^{2}
\end{gathered}
$$

in which Poisson's ratio has been taken as $1 / 3$.

In [4] it was shown that a good approximation to the linear response following arbitrary radial impulse is obtained by neglecting tangential inertia. We extend this approximation here to the deviation from the basic response, With this from (7a) and ( $7 \mathrm{~b})$

$$
\begin{aligned}
& U_{m n}=\frac{C_{5} C_{2}-C_{3} C_{4}}{C_{1} C_{4}-C_{2}{ }^{2}} w_{m n} \\
& v_{m n}=\frac{C_{2} C_{3}-C_{1} C_{5}}{C_{1} C_{4}+C_{2}{ }^{2}} w_{m n}
\end{aligned}
$$

Introducing (9) and. W from (5) into (7c) then gived

$$
w_{m n}+(\Omega+\mu \sin T) w_{m n}=0
$$

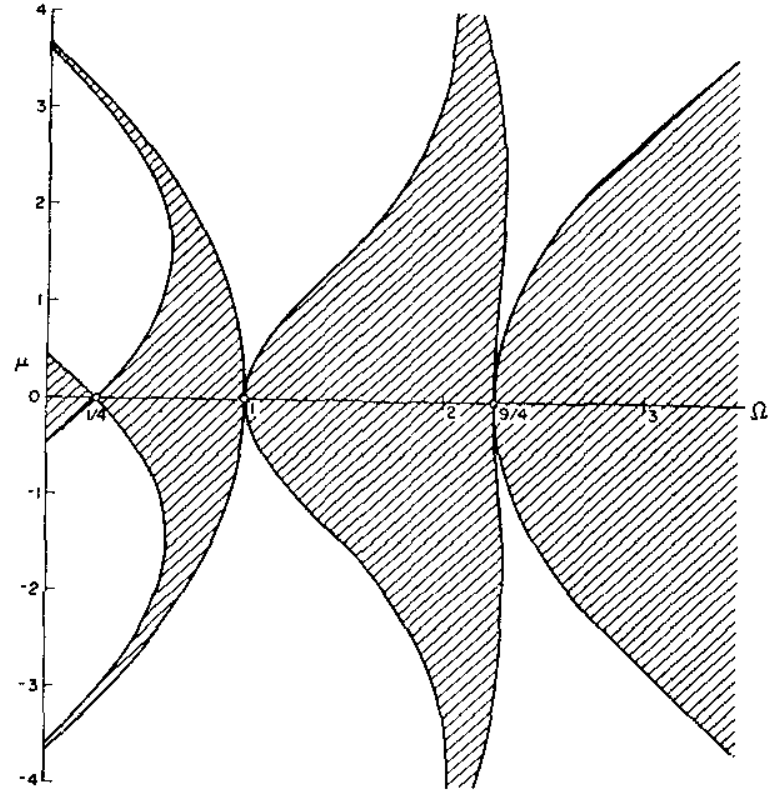

Fig. 2 Mathieu Stability Diagram

where

$$
\begin{aligned}
& \Omega=c_{6} \frac{c_{5}^{2}}{C_{4}}+\frac{\left(c_{3} C_{4}-C_{2} c_{5}\right)^{2}}{c_{4}\left(C_{2}^{2}-C_{1} C_{4}\right)} \\
& \mu=\frac{\sigma_{0}}{c}\left[n^{2}+\frac{m^{2} \pi^{2}}{3}\left(\frac{a}{l}\right)^{2}\right]
\end{aligned}
$$

Equation (10) is Mathieu's equation, whose properties and solutions a re exten'sively recorded such as in [5]. The chaxacter of the solution is determined by the parameters $\Omega$ and $\mu$. Figure 2 shows the Mathieu stability diagram. If a point $(\Omega, \mu)$ falls within a shaded region, the solution is bounded or stable; if the point falls within an unshaded region, the solution exhibits exponential growth. For a fixed value of $\mu$ the instability zones become increasingly narrow as $\Omega$ increases. In the present problem the amplitude of a given mode will remain perturbational in size whenever its corresponding parameter point falls into a stable region. But significant growth may be expected in a mode whose parameter point falls in an unstable region,

In a given problem we must locate the parameter points for each pair $(m, n)$. For larger values of either integer the parameter points fall in the predominantly stable region. Thus in generafti. we may confine our attention to the first unstable zone. 


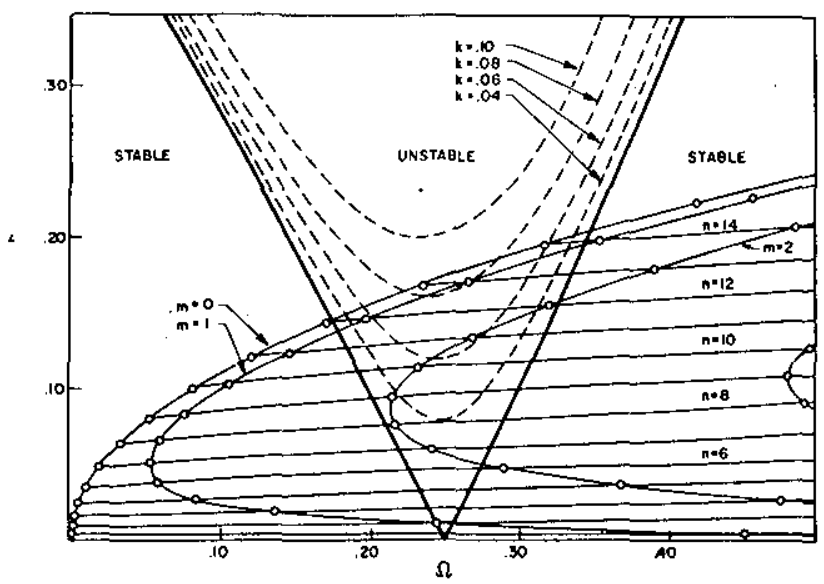

Fig. 3 Stability Diagram for $\mathrm{v}_{\mathrm{o}} / \mathrm{c}=10^{-3}, \mathrm{a} / \mathrm{l}=1, \mathrm{a} / \mathrm{h}=100$

If $m$ and $n$ are treated as continuous variables, eqiations (11a) and (11b) may be viewed as the parametric representation of a doubly infinite fainily of curves. The intersection of a curve as sociated with an integer value of $m$ with the curve associated with an integer value of $n$ is the parametric point for the pair $(m, n)$. The set of curves for a particular geometry $(a / \ell=1, a / h=$ $100)$ and initial velocity $\left(v_{0} / c=10^{-3}\right)$ is shown in Finure 3. A relatively large number of points fail in the unstable zone. The points on the $m=0$ curve coincide with the plane strain solution given in $[1]$.

Figure 4 shows the corresponding results for $\mathrm{a} / \ell=1 / 2$ and $\mathrm{a} / \mathrm{h}=100$. If the shell is lengthened, more points move into the unstable zone from the right, which can be seen by comparing Figures 3 and 4. As the length becomes arbitrarily large, for each $n$ the points for all values of $m$ coalesce to the $m=0$ point.

The examples shown are typical for a wide range of geometries. It is evident that the basic response is likely to be unstable since usually at least one parameter point will fall in the unstable zone. Generally a relatively large number of modes will be associated with the instability, the number increasing with increasing length of the shell.

Not all of these modes, however, will have a significant effect on the long term response. For the modes with parameter points in the unstable zone, the Mathieu solution is of the form $\phi(\tau)$. expk $T$ where $\phi(\tau)$ is periodic. Iso-k curves are shown dashed in Figures 3 and 4 . It is expected that the modes $w$ ith the higher growth rates will dominate the response. Thus many of the unstable modes may be neglected based upon a comparison of their initial growth rates. The accuracy obtained For a given choice of modes is discussed in the

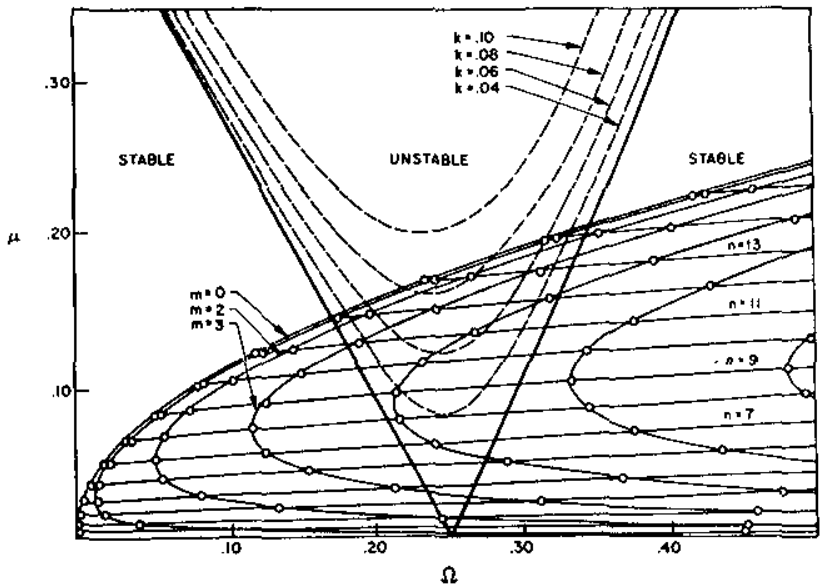

Fig. 4 Stability Diagram for $v_{0} / c=10^{-3}, a / \ell=1 / 2, a / h=100$

next section.

\section{Long Term Response}

To obtain the finite time response, the nonlinear terms in the governing equations for the Fourier coefficients must be retained. It is necessary, however, to consider only modes which undergo significant growth. Modes exhibiting initial growth were identified in the preceding stability analysis. Of this group the modes with the higher growth rates will dominate the long term response.

Since not all possible "unstable" modes will be included in the analysis, the accuracy of the solution will be ascertained by computing the total energy of all participating modes and comparing it to the kinetic energy initially imparted to the shell. Also, we require the energy of the slowest growing of the selected modes to be small compared with the initial energy. This assures that the excluded modes have negligible effect upon the solution.

The nonlinear equations have been integrated numerically for a number of geometries and different initial imperfections. Some typical results are given here.

Example 1

In this case $a / l=1.0$ and $a / h=100$. The stability diagram in the vicinity of $\Omega=1 / 4$ is shown in Figure 3. Although ten modes are sus. ceptible to excitation, only the four fastest growing modes, $(0,13),(1,13),(0,14)$, and $(2,11)$ are included in the analysis.

The initial conditions used are

$$
\frac{\dot{w}_{00}(0)}{a}=\frac{v_{0}}{c}
$$




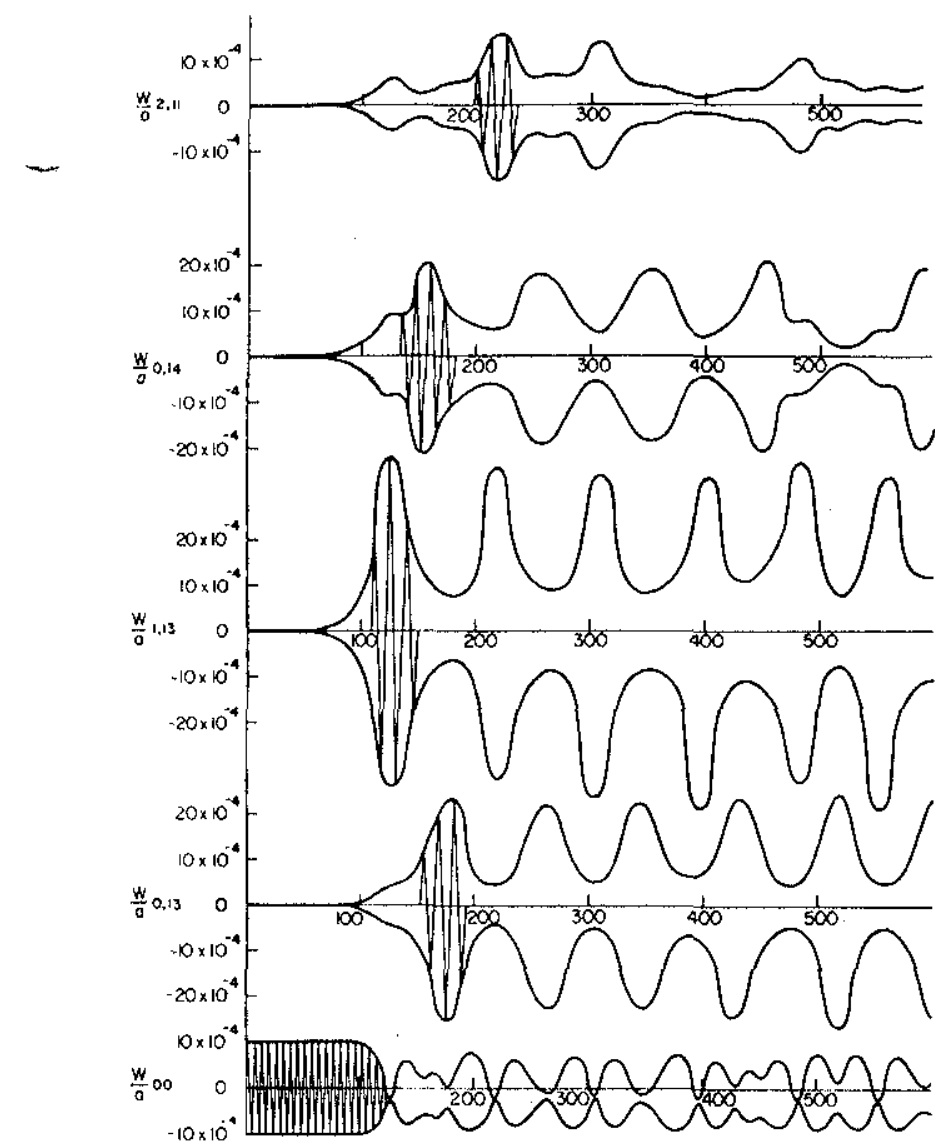

Fig. 5 Displacement Coefficients vs. Time for Example 1

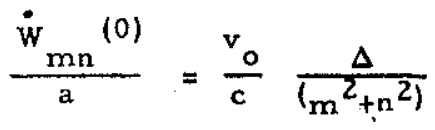

in which $\Delta<<1$.

The numerical results obtained for $v_{0} / c=10^{-3}$ are shown in Figure 5. The response is characterized by cyclic exchange of energy between the various modes. The largest amplitude is attained by mode $(1,13)$; this is also the firat mode to reach its maximum value. Clearly the displacements associated with the finite time response are considerably in excess of those associated with the unperturbed motion,

The ratio of the total energy in each mode to the initial kinetic energy of the shell is shown in Figure 6. The mode with the slowest growth rate, (2,11), participates with almost negligible energy during the early exchanges and never contains more than $15 \%$ of the total energy for the range of tine considered. The maximum difference between the total energy of the modes considered and the original kinetic energy never exceeded $1 / 2 \%$.

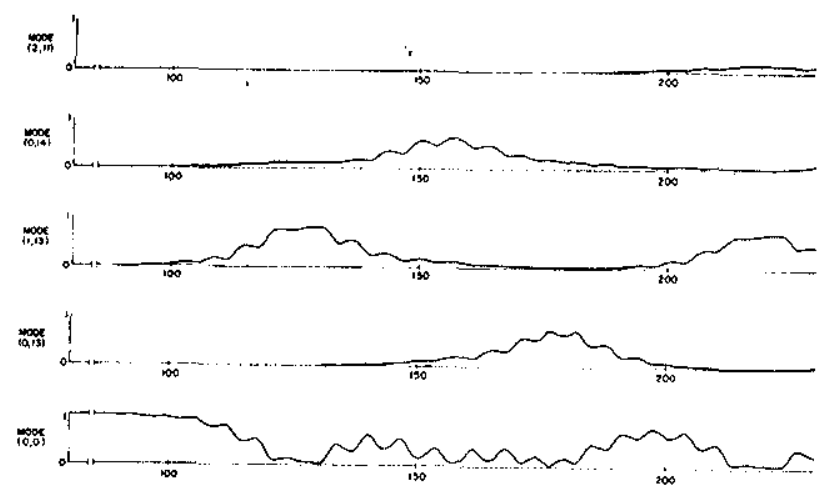

Fig. 6 Energy Ratios vs. Time for Example 1

\section{Example 2}

A second perturbation is considered with the same geometry as in Example 1. The initial radial velocity distribution is taken as a constant perturbed by a parabolic variation. Thus

$$
\begin{array}{rlrl}
\frac{w}{a}(x, \theta, 0) & =\frac{v}{c}\left[1+\Delta\left(\frac{x^{2}}{l}\right)^{2}\left(1-\frac{4 \theta^{2}}{l^{2}}\right)\right]-\frac{\pi}{2}<\theta<\frac{\pi}{2} \\
& =\frac{v_{0}}{c} & \frac{\pi}{2}<\theta<\frac{3 \pi}{2}
\end{array}
$$

The numerical results, again computed for $v_{0} / c=10^{-3}$, are shown in Figure 7. Although the mode $(0,14)$ now reaches its maximum magnitude first, the largest amplitude is attained by $(1,13)$ a few cycles later when essentially all of the energy in the basic motion has been extracted.

Comparison of the two examples shows that the maximum amplitude attained by a given mode is rather insensitive to changes in the perturbation. Thus the nature of initial imperfections will not alter the dominant modes in the long term response. They may, however, alter the relative phasing of the dominant modes. In the present example the fact that the mode $(0,14)$ reaches its maximum first can be attributed to the relative size of the perturbations. In Example l the perturbations received by each mode were of comparable magnitude, while in Example 2 the initial value for $10,14)$ was approximately 15 times as strong as that for $(1,13)$. Since the displacement at any instant depends upon the relative phase of the various modes, the actual motion is quantatatively sensitive to the initial values of the dominant modes.

Example 3

If the shell is lengthened, more modes are susceptible to excitation. For example, the stability diagram in the vicinity of $\Omega=1 / 4$ for $a / \ell=1 / 2$ is shown in Figure 4. Using the parabolic 

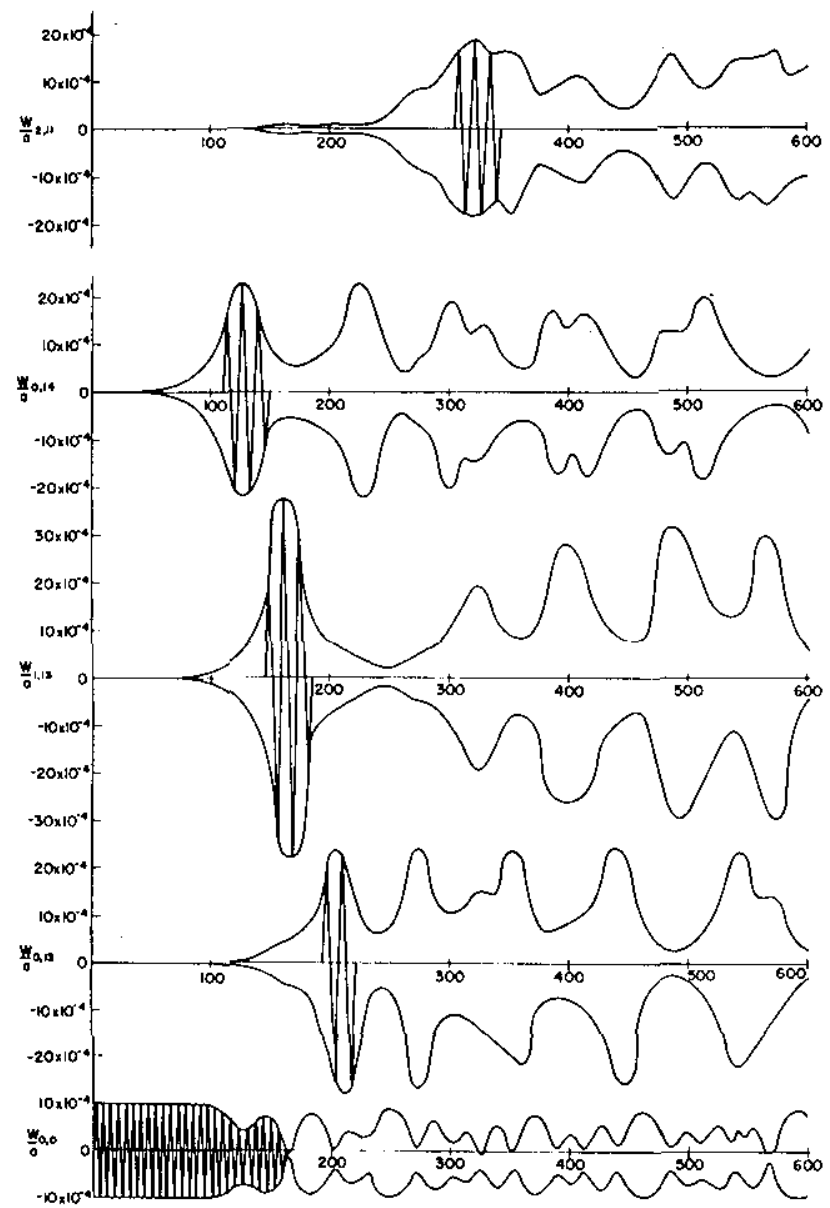

Fig. 7 Displacement Coefficients $\times 8$. Time for Example 2

perturbation of Example 2, the nonlinear equations for the six fastest growing modes were integrated with $v / c=10^{-3}$. The results are qualatatively similar to the previous example with the long term motion being dominated by the same three modes. This confirms the expectation that the character of the finite time response is determined more by the initial growth rates than by the number of modes susceptible to excitation.

\section{Axial and Circumferential Normal Stress}

The large displacements which develop in the finite time response of the shell are accompanied by comparably magnified stresses. In many applications the stress level would be of prime importance.

For a state of plane stress the normal stress in the circumferential direction is

$$
\begin{aligned}
\sigma_{\theta} & =\frac{E}{\left(1-v^{2}\right)} \sum_{i=0}^{\infty} \sum_{j=0}^{\infty}\left\{\frac{w_{i j}}{a}\left[1-\frac{z}{a}\left(1-j^{2}-i^{2} v \frac{\pi^{2} a^{2}}{l^{2}}\right)\right]\right. \\
& \left.+j \frac{v_{i j}}{a}+i v \pi\left(\frac{a}{l}\right) \frac{U_{i j}}{a}\right\}
\end{aligned}
$$
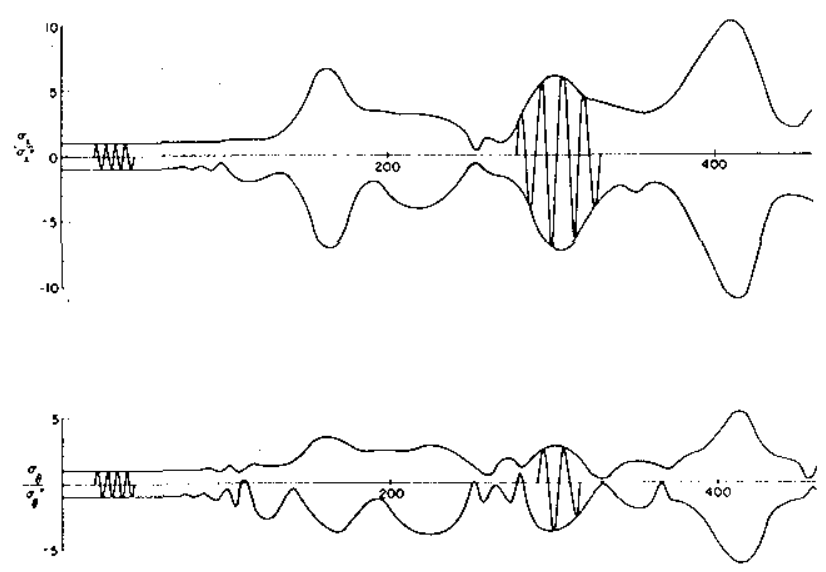

Fig. 8 Stress Ratios v8. Time for Example 2

The axial stress is

$$
\begin{aligned}
& \sigma_{x}=\frac{E}{\left(1-v^{2}\right)} \sum_{i=0}^{\infty} \sum_{j=0}^{\infty}\left\{\frac{w_{i j}}{a}\left[v-\frac{z}{a}\left(v-v^{2}-i^{2} \frac{\pi^{2} a}{l^{2}}\right)\right]\right. \\
&\left.\left.+i \pi \mid \frac{a}{l}\right) \frac{U_{l j}}{a}+j v \frac{v_{l j}}{a}\right\}(14 b)
\end{aligned}
$$

The stress components at a typical point, $x \times 0$, $\theta=0, z=h / 2$, are computed for Example 2. For the unperturbed or basic motion of the shell, the maximum circumferential stress is

$$
\sigma_{\theta}^{*}=\frac{E v_{o}}{\left(1-v^{2}\right)_{c}}
$$

The amplitude of the axial stress for the fundamental motion is

$$
\sigma_{x}^{*}=\frac{v E v_{0}}{\left(1-v^{2}\right) c}
$$

Equations (14) are written as finite sums for the fundamental and excited modes. The ratios of these stresses to the maximum values of the basic motion are shown in Figure 8 . In this example the circumferential and longitudinal stress ratios reachextreme values of 5 and 10 respectively. Maximum stress does not necessarily occur with the first significant energy exchange, but rather at a later time when the modes dominating the finite time response tend to be in phase.

\section{Conclusions}

The stability of the motion resulting from the application of a uniform radial impulse to a finite length cylindrical shell has been analyzed. It has been shown that an unstable response is characterized' by the exitation of flexural modes which exchange energy with the basic motion in a cyclic 
manner. The possibility for such a response is increased both by lengthening the shell and decreasing its thickness. A criterion has been extablished for the identification of the excited modes.

The growth rates of the perturbed modes can be determined from a short term analysis, Generally the long term behavior is dominated by the flexural modes with the higher growth rates. The long term response is sensitive to changes in the initial perturbations received by the se high growth rate modes, but is insensitive to changes in the initial values of the slow growth modes.

Finally it has been shown that if flexural motion is excited, it gives rise to atresses that are far in excess of those associated with the basic motion of the shell.

\section{Acknowledgements:}

The work reported in this paper was partially supported by the National Science Foundation under Grant No. GK-1251 with the University of Michigan.

\section{Reference $B$}

1: Goodier, J. N. and Mcivor, 1. K., "The Elastic Cylindrical Shell Under Nearly Uniform Radial Impulse," J. Appl. Mech., Vol. 31, No. 2, June 1964, pp, 259 266.

2. Mclvor, I. K, and Sonstegard, D, A., "Axisymmetric Response of a Closed Spherical Shell to a Nearly Uniform Radial Impulse," : J. Acoust. Soc. Am., Vol. 40, No. 6, Dec. 1966, Pp. 1540-1547.

3. Bieniek, M. P., Fan, T. C., and Lackman, L. M., "Dynamic Stability of Cylindrical Shells," A LAA Journal, Vol. 4, No. 3, Mar. 1966. pp. 495-500.

4. Lovell, E. G., and McIvor, I.K., "The Cylindrical Shell of Finite Length Under A Nearly Uniform Radial Impulse", Technical Report No. 08371-1-T, University of Michigan, June, 1967.

5. McLachlan, N. W., Theory and Application日 of Mathieu Functions, Dover Publications, Inc., New York, 1964

\section{Appendix}

The material of the shell is assumed to be isctropic and linearly elastic with modulus of elisticity $E$ and Poisson's ratio $v$. The strain enurgy in a form appropriate for the nonlinear problem considered here has been derived in [4]. It is

$$
U=U_{m}+U_{b}
$$

where

$$
\begin{aligned}
u_{m}^{\prime} & =\frac{k}{2} \int_{0}^{2 \pi} \int_{0}^{l}\left[\left(a u_{x}^{2}+\frac{v_{\theta}^{2}}{a}+\frac{w^{2}}{a}\right.\right. \\
& \left.+2 \frac{w v_{\theta}}{a}+a u_{x} w_{x}^{2}+\frac{v_{\theta}^{w} w_{\theta}^{2}}{a}+\frac{w w_{\theta}^{2}}{a}\right) \\
& +v\left(2 u_{x} v_{\theta}+2 u_{x} w+\frac{u_{x} w_{\theta}^{2}}{a}+v_{\theta} w_{x}^{2}+w w_{x}^{2}\right) \\
& +(1-v)\left(\frac{u_{\theta}^{2}}{2 a}+\frac{a v_{x}^{2}}{2}+u_{\theta} v_{x}\right. \\
& \left.\left.+\frac{u_{\theta} w_{x} w_{\theta}}{a}+v_{x} w_{x} w_{\theta}\right)\right] d x d \theta
\end{aligned}
$$

$$
\begin{aligned}
& \mathrm{u}_{\mathrm{b}}=\frac{\mathrm{D}}{2} \int_{0}^{2 \pi} \int_{0}^{l}\left[\left\langle-2 \mathrm{u}_{\mathrm{x}} \mathrm{w}_{\mathrm{xx}}+\mathrm{aw} \mathrm{w}_{\mathrm{xx}}^{2}\right.\right. \\
& +\frac{w_{\theta \theta}^{2}}{a^{3}}+2 \frac{{ }^{w} \theta \theta}{a^{3}}+2 \frac{{ }^{w} \theta \theta^{w}{ }^{2}}{a^{4}} \\
& \left.-w_{\mathbf{x x}} \mathrm{w}^{2} \mathbf{x}\right)+v\left(2 \frac{\mathrm{w}_{\mathbf{x x}} \mathbf{w}^{\mathrm{w}} \theta \theta}{\mathrm{a}}-2 \frac{\mathrm{v}^{\mathrm{w}} \mathbf{x x}}{\mathrm{a}}\right. \\
& \left.+\frac{{ }^{w} x^{w} w_{\theta}}{a^{2}}\right)+(1-v)\left(\frac{u_{\theta} w_{x \theta}}{a^{2}}-3 \frac{\mathbf{x}^{w} \mathbf{x} \theta}{a}\right. \\
& \left.\left.+2 \frac{w^{2} x \theta}{a}+\frac{w_{x \theta} x^{w} \theta}{a^{2}}\right)\right] d x d \theta
\end{aligned}
$$

and

$$
K=E h /\left(1-v^{2}\right), \quad D=E_{h^{3}} / 12\left(1-v^{2}\right)
$$

In $(A-2)$ and $(A-3)$ a subscript denotes partial differentiation with respect to the coordinate.

With $\rho$ for mass density the kinetic energy is

$$
T=\frac{\rho h a}{2} \int_{0}^{2 \pi} \int_{0}^{l}\left[\left(\frac{\partial u}{\partial t}\right)^{2}+\left(\frac{\partial v}{\partial t}\right)^{2}+\left(\frac{\partial w}{\partial t}\right)^{2}\right] d x d \theta
$$

When the displacement representation (1) is introduced into $(A-1)$ and $(A-5)$, the coefficients can be considered as generalized coordinates. The corresponding Lagrange equation for the basic response $W_{\text {oo }}$ is 


$$
\begin{aligned}
& \frac{\ddot{w}_{o 0}}{a}+\frac{w_{o o}}{a}+\frac{1}{4}\left[\sum_{j=1}^{\infty} j^{2}\left(\frac{w_{o j}}{a}\right)^{2}+\frac{\pi^{2}}{3}\left(\frac{a}{l}\right)^{2} \sum_{i=1}^{\infty} i^{2}\left(\frac{w_{i o}}{a}\right)^{2}\right. \\
& \left.\left.\frac{1}{2} \sum_{i=1}^{\infty} \sum_{j=1}^{\infty} j^{2}\left(\frac{w_{i j}}{a}\right)^{2}+\frac{\pi^{2}}{6} \mid \frac{a}{l}\right)^{2} \sum_{l=1}^{\infty} \sum_{j=1}^{\infty} i^{2}\left(\frac{w_{i j}}{a}\right)^{2}\right]=0
\end{aligned}
$$

$(A-6)$

in which the dot denotes differentiation with respect to the dimensionless time $T$ defined by equation (3).

The Lagrange equation for $\mathrm{W}_{\mathrm{mn}}$ is

$$
\begin{aligned}
& \frac{\ddot{w}_{m n}}{a}+\frac{w_{m n}}{a}\left[1+\frac{\pi^{4} m^{4}}{12}\left|\frac{h}{a}\right|^{2}\left(\left.\frac{a}{l}\right|^{4}+\frac{n^{4}}{12}\left(\frac{h}{a}\right)^{2}-\frac{n^{2}}{6}\left(\frac{h}{a}\right)^{2}+\frac{\pi^{2} m^{2} n^{2}}{6}\left(\frac{h}{a}\right)^{2}\left(\frac{a}{l}\right)^{2}\right]\right. \\
& \left.\left.+U_{m n}\left[\frac{\pi m}{3} \mid \frac{a}{l}\right)+\frac{\pi^{3} m^{3}}{12} \mid \frac{h}{a}\right)^{2}\left(\frac{a}{l}\right)^{3}-\frac{\pi m n^{2}}{36}\left(\frac{h}{a}\right)^{2}\left(\frac{a}{l}\right)\right] \\
& +\frac{v_{m n}}{a}\left[n+\frac{\pi^{2} m^{2} n}{g}\left(\frac{h}{a}\right)^{2}\left(\frac{a}{l}\right)^{2}\right]+\left(\frac{w_{o n} w_{m o}}{a^{2}}+\frac{w_{o 0} w_{m n}}{a^{2}}\right)\left(n^{2}+m^{2} \frac{\pi^{2} a^{2}}{3 l^{2}}\right) \\
& +\frac{1}{2}\left[\sum_{i=1}^{\infty} \frac{w_{i o} w_{m+i, n}}{a^{2}}\left(n^{2}+\frac{\pi^{2} m^{2} a^{2}}{3 l^{2}}+\frac{\pi^{2} i m a^{2}}{3 l^{2}}+\frac{\pi^{2} i^{2} a^{2}}{3 l^{2}}\right)\right. \\
& +\sum_{i \neq 1}^{m-1} \frac{w_{i 0} w_{m-i, n}}{a^{2}}\left(n^{2}+\frac{\pi^{2} m^{2} a^{2}}{3 l^{2}}-\frac{\pi^{2} i m a^{2}}{3 l^{2}}+\frac{\pi^{2} i^{2} a^{2}}{3 l^{2}}\right) \\
& \left.+\sum_{i=1}^{\infty} \frac{w_{i n} w_{i+m, 0}}{a^{2}}\left(n^{2}+\frac{\pi^{2} m^{2} a^{2}}{3 l^{2}}+\frac{\pi^{2} i m a^{2}}{3 l^{2}}+\frac{\pi^{2} i^{2} a^{2}}{3 l^{2}}\right)\right] \\
& +\frac{1}{4}\left\{\sum_{i=1}^{\infty} \sum_{j=1}^{\infty} \frac{w_{i j} w_{i+m, j+n}}{a^{2}}\left[j^{2}+j n+n^{2}+\frac{\pi^{2} a^{2}}{3 k^{2}}\left(i m+i^{2}+m^{2}\right)\right]\right. \\
& +\sum_{i=1}^{\infty} \sum_{j=n+1}^{\infty} \frac{w_{i j} w_{i+m, j-n}}{a^{2}}\left[j^{2}-j n+n^{2}+\frac{\pi^{2} a^{2}}{3 l^{2}}\left(i m+i^{2}+m^{2}\right)\right] \\
& +\sum_{i=1}^{\infty} \sum_{j=1}^{n-1} \frac{w_{i j} w_{i+m, n-j}}{a^{2}}\left[j^{2}-j n+n^{2}+\frac{\pi^{2} a^{2}}{3 \ell^{2}}\left(i m+i^{2}+m^{2}\right)\right] \\
& \left.+\sum_{i=1}^{m-1} \sum_{j=1}^{\infty} \frac{w_{i j} w_{m-i, n+j}}{a^{2}}\left[j^{2}+j n+n^{2}+\frac{\pi^{2} a^{2}}{3 l^{2}}\left(-i m+i^{2}+m^{2}\right)\right]\right\} \\
& +\frac{1}{8}\left\{\sum _ { i = 1 } ^ { m - 1 } \sum _ { j = 1 } ^ { n - 1 } \frac { w _ { i j } w _ { m - i , n - j } } { a ^ { 2 } } \left[\left(n j+j^{2}\right)+\frac{\pi^{2} a^{2}}{3 l^{2}}\left(m i+i^{2}\right)-\frac{h_{n j}^{2}}{6 a^{2}}(n-j)(2 j-n)\right.\right.
\end{aligned}
$$

$(\mathrm{A}-7)$ 
$+\frac{m \pi^{4}}{12}\left(\frac{a}{l}\right)^{2}\left(\frac{h}{a}\right)^{2} i(m-i)(m-2 i)+\frac{\pi^{2}}{18}\left(\frac{h}{a}\right)^{2}\left(\frac{a}{l}\right)^{2}[n i(m-i)(n-2 j)$

$+m j(n-j)(m-2 i)]\}=0$

The equations for the other coefficients are structurally similar to $(A-7)$ and for brevity will not be reproduced here. 